\title{
Intermediate-band photometry of a rich cluster of galaxies: A2218
}

\author{
K. Rakos ${ }^{1}$, D. Dominis ${ }^{2}$, and S. Steindling ${ }^{3}$ \\ 1 Institute for Astronomy, University of Vienna, Austria \\ 2 Faculty of Geodesy, University of Zagreb, Croatia \\ 3 Wise Observatory, School of Physics and Astronomy, Tel Aviv University, Israel
}

Received 23 August 1999 / Accepted 19 December 2000

\begin{abstract}
This paper presents four color intermediate-band photometry of the cluster A2218 carried out using the Wise Observatory $1 \mathrm{~m}$ telescope. A2218 is one of the richest clusters in the Abell catalogue, with richness class 4. We find that A2218 has an unusually low fraction of blue galaxies for its redshift $(z=0.171)$, and is populated mainly by E/S0 galaxies, while starburst systems are very scarce. The few starbursting galaxies present populate the faint end of the cluster luminosity function, close to the limiting magnitude of our observations. The difference between A2218 and other clusters at similar redshifts is very remarkable, underlining the influence of cluster richness on the evolution of its member galaxies.
\end{abstract}

Key words. galaxies: clusters: individual: A2218 - galaxies: evolution - galaxies: fundamental parameters galaxies: photometry

\section{Introduction: Evolution of cluster galaxies}

Early expectations from galaxy evolution models were that strong color evolution would only be visible in galaxies at $z \geq 1$ and thus would be redshifted out of the realm of optical telescopes. However, two facts were discovered in the last twenty years: (1) the sharp rise in the fraction of blue cluster galaxies at intermediate redshifts (Butcher \& Oemler 1984, hereafter B\&O), called the Butcher-Oemler effect, and (2) the excess of low-luminosity blue galaxies in the field population at intermediate redshifts (Koo 1986; Tyson 1988). These showed that galaxy evolution is more than simple color changes calculated for passively evolving stellar populations. In an earlier paper (Rakos \& Schombert 1995), we used rest-frame four-color photometry (the Strömgren system) to explore the color evolution of 17 galaxy clusters for $0.2 \leq z \leq 0.9$. In that paper, the red population was shown to follow closely the passive evolution models for a single burst population, with an epoch of galaxy formation near $z=5$. On the other hand, the fraction of blue galaxies showed a dramatic increase with redshift, reaching $80 \%$ at $z=0.9$. In earlier publications, when the high- $z$ observations were not yet available, the authors interpreted this as the rapid change of star forming disk galaxies into S0s.

However, in the high redshift range of our study, the majority of the clusters galaxy population is involved in some amount of star formation. Since the results for the

Send offprint requests to: K. Rakos,

e-mail: karl.rakos@chello.at red (elliptical) population are consistent - at all redshifts with a formation epoch of $z=5$ followed by passive evolution, this means that all other galaxies (S0s, spirals, and irregulars) are star forming at $z=0.9$. The transformation of so many galaxies into S0s being inconsistent with present-day morphological cluster statistics, some other scenario must be invoked. This could be either a slow tapering off of the star formation by gas depletion or stripping, or else the demise of many members of the blue population must take place by the present epoch, either by complete destruction or by reduction to the luminosity of dwarf galaxies.

Using the Strömgren $m z=(v z-b z)-(b z-y z)$ color index, Rakos et al. (1996) and Rakos et al. (1997) have shown that many of these blue galaxies have strong starburst or post-starburst signatures. Inspection of highresolution images (Rakos et al. 1996) revealed that such starburst activity, as traced by the $m z$ index, correlates with mergers and close pair interactions in the local Universe. This is also consistent with the findings of Dressler et al. (1994a, b) for A851 (at $z=0.402$ ). This suggests that a fraction of the blue population in ButcherOemler clusters is the progenitor of the present day S0s in rich clusters, under the assumption that S0s are the outcome of merger events.

Tidal interactions may well explain the remainder of the high fraction of starburst galaxies: these would have their origin in gas-rich dwarf galaxies undergoing a short but intense, tidally induced starburst. It should be noted however, that the orbits of cluster galaxies are primarily 
radial, and the typical velocities are high. This makes any encounter with another galaxy short-lived, with too little impulse being transferred, than would be required to shock molecular clouds into a nuclear starburst. Recently, a new mechanism for tidally induced star formation has been proposed. This phenomenon, called galaxy harassment (Moore et al. 1996), emphasizes the influence of the global cluster tidal field and the cumulative effect of rapid impulse encounters on disk galaxies. These two processes conspire not only to raise the luminosity of cluster spirals, but also to considerably increase the visibility and hence detectability of low surface brightness objects. Galaxy harassment predicts that galaxies in the cores of clusters will have globally older stellar populations than galaxies at the cluster edges. In terms of star formation history, this is exactly what has been demonstrated in A2317 (Rakos et al. 1997): the blue population is primarily located in the outer two thirds of the cluster.

The model also predicts, that the final stage of harassed galaxies will be a dwarf spheroidal with a fading signature of recently experienced starburst activity. Evidence for such objects is accumulating in the literature: There is evidence that dwarfs in the Local Group possess young stellar populations (Gallagher \& Wyse 1994). Nucleated dwarfs in Virgo, Fornax and Coma show spectroscopic and photometric features indicative of young stars (Held \& Mould 1994; Donas et al. 1995; Caldwell \& Rose 1998), and so do other, non-nucleated dwarf irregulars in Virgo (Almoznino \& Brosch 1998a, b; Heller et al. 1999). The rich populations of dwarf galaxies now being discovered in clusters (Sandage et al. 1985; Driver et al. 1994; de Propris et al. 1995; Phillipps et al. 1998) may represent the remnants of these Butcher-Oemler objects.

But harassment is not the only mechanism, by which dwarf and/or low surface brightness objects can account for the past abundance of blue galaxies: the excess in blue counts of field galaxies with respect to no-evolution models has also been attributed to a population of dwarf galaxies at intermediate redshift undergoing bursts of star formation (Tyson 1988). Hogg \& Phinney (1997) have presented a model in which field dwarfs undergo a series of star formation bursts. This was verified for Virgo irregulars by fitting evolutionary models to $U B V R I$ and $\mathrm{H}_{\alpha}$ photometry (Almoznino \& Brosch 1998b). Since the burst luminosity remains high in red passbands for long periods, Hogg \& Phinney (1997) propose that this generates a steep luminosity function for dwarfs, whose slope increases with wavelength. Also, Silk et al. (1987) have argued that dwarfs switch star formation on and off, possibly in a feedback effect. During "high" phases, spheroidals would appear as irregulars, before returning to quiescence. Such objects would be excellent candidates for the ButcherOemler systems of Koo et al. (1997) and Oemler et al. (1997). Once the starburst is over, such objects would fade in luminosity and surface brightness below the limits of detectability (Abraham et al. 1996).

Whatever the mechanism, the first observational evidence for the role of dwarf galaxies in distant clusters has been found in A2317 (Rakos et al. 1997). We have shown that the blue galaxies dominate at the bright and faint extremities of the cluster luminosity function. The family of faint blue galaxies contains a larger fraction of starbursting galaxies than the bright blue galaxies. The bright galaxies have finished their starburst phase, if any, earlier in the past, and show only low level but steady production of new stars.

\section{The case of $A 2218$}

The cluster A2218 exhibits many extreme properties: it is one of the richest clusters in the ACO catalogue (Abell et al. 1989) with a richness class 4 (for comparison, Coma has $R=3$ ). It contains a large $\mathrm{cD}$ galaxy in its center. The galaxy has a very low central surface brightness and an envelope extending over more than $180 \mathrm{kpc}$ (Pello-Descayre et al. 1988). An analysis of the observations by Butcher et al. (1983) and Butcher \& Oemler (1984) gives a concentration parameter $C=0.59$, one of the largest in their sample. ( $C$ is the ratio of radii containing $20 \%$ and $60 \%$ of the counts in their magnitude range respectively.) Birkinshaw \& Hughes (1994) measured a strong SunyaevZeldovich effect. A2218 has also been detected in X-rays (David et al. 1993). The peak of the X-ray surface brightness distribution is coincident with the location of the $\mathrm{cD}$ galaxy (McHardy et al. 1990; Yamashita 1995). A2218 has a central velocity dispersion of $1370 \mathrm{~km} \mathrm{~s}^{-1}$ (Le Borgne et al. 1992). Deep optical images of the cluster have revealed numerous arcs and arclets (Pello et al. 1992). Their location and morphology suggest that the bulk of the cluster mass is associated with the galaxy concentration surrounding the $\mathrm{cD}$ galaxy. The photometric and spectroscopic study of the cluster center suggests that the cluster consists in fact of two galaxy concentrations, of which one is centered about the cD galaxy. This is confirmed by the lensing analysis of Kneib et al. (1995). The clumpy X-ray emission then appears as a direct consequence of the ongoing merging of these two sub-units, as the gas has not yet reached equilibrium (Kneib et al. 1995). Thus, A2218 appears to be in a merger state, showing a bimodal structure. Investigating how such an extreme object fits into the general picture described above can give us insight on the trigger behind the Butcher-Oemler effect.

In this paper we describe observations of A2218 with the specific goal of understanding the nature of $\mathrm{B} \& \mathrm{O}$ galaxies. In Sect. 2 we describe the observations and their reductions. The results are presented in Sect. 3, and we conclude with a summary and discussion in Sect. 4 .

\section{Observing and analysis techniques}

Observations were made in April 1997 with the Wise Observatory's f/ 7 1m telescope and TEK $1024 \times 1024$ CCD camera using rest frame Strömgren uvby filters appropriate for A2218, called $u z, v z, b z$ and $y z$ hereafter. Each of these filters is approximately $200 \AA$ wide and was designed specially to match the cluster redshift $(z=0.171)$ in 
order to maintain a rest-frame color system. The CCD chip was binned 2 by 2 to $1.4^{\prime \prime} /$ pixel, appropriate to the actual seeing of $2^{\prime \prime}$. A $12^{\prime}$ square field of view was imaged for this investigation. Four exposures of $900 \mathrm{~s}$ in each filter were co-added. Colors and magnitudes were measured on the co-added images using standard IRAF procedures and are based on metric apertures set at $32 \mathrm{kpc}$, for cosmological parameters of $H_{0}=50 \mathrm{~km} \mathrm{~s}^{-1} \mathrm{Mpc}^{-1}$ and $q_{0}=0$. Objects were selected by eye in each of the four final images, and only those visible in all four bands were retained for photometry using the IRAF APPHOT package. The photometric calibration used spectrophotometric standards from Massey et al. (1988) combined with tabulated filter transmission curves. Instrumental magnitudes were converted to the Strömgren system using our standard star observations via the formalism that has been described in detail in Rakos et al. (1988). Foreground Galactic reddening in the direction of A2218 is negligible (Dickey \& Lockman 1990). Our typical errors were $0.05 \mathrm{mag}$ in color at the bright end of the sample $\left(m_{\mathrm{AB}}(5500 \AA) \simeq 16\right)$ and $0.1 \mathrm{mag}$ at the faint end $\left(m_{\mathrm{AB}}(5500 \AA) \simeq 19\right)$.

\subsection{Identification of cluster members}

Cluster population statistics derived from photometry have often been criticized for contamination by stars as well as by fore- and background galaxies. Since our filters are "tuned" to the redshift of the cluster, we can use colors to discriminate between stars, foreground, and background objects in a very efficient manner. We calculated synthetic Strömgren colors of a compilation of 143 spectra of nearby galaxies covering all possible types, to demonstrate that galaxies observed in their rest-frame Strömgren bands are confined to a very limited region of the three-dimensional color-space subtended by the four bands (Steindling et al. 2001). Color differences due to the mismatch between object and filter redshift are much larger than those among galaxies of different type viewed in their rest-frame Strömgren bands. Since we are using real galaxies as templates and not models, we need not worry about dust rendering the cluster galaxy colors unrecognizable, because our nearby galaxy sample includes very dusty galaxies too.

Simulating the appearance of stars, by convolving the spectra of the lcb97 stellar library (Lejeune et al. 1997, 1998) with our filter transmission curves, we showed that most stellar types are easily recognized and can be discarded, based on their "absurd" colors. The case of foreand background galaxies is very similar: except for foreground starburst systems, the mismatch between their redshift, and that of the target cluster (for which the filters are designed) causes the colors to be significantly different from any possible cluster member. The efficiency of this rejection mechanism has been demonstrated in Steindling et al. (2001). Estimates of the number of remaining unrecognizable field stars and galaxies were made based on the Bahcall-Soneira galaxy model for Galactic stars (Bahcall 1986) and the Canada-France redshift survey for galaxies (Lilly et al. 1995; Crampton et al. 1995) and shown to be completely negligible in most cases.

Note that our filters are narrow enough to obtain data of sufficient spectral resolution for identification and classification purposes, but sufficiently wide for the cluster's velocity dispersion not to affect the colors.

\subsection{Galaxy classification}

Based on the aforementioned high resolution, high $\mathrm{S} / \mathrm{N}$ spectral templates compiled from the catalogs of Gunn \& Oke (1975), Yee \& Oke (1978), Kennicutt (1992), de Bruyn \& Sargent (1978) and Ashby et al. (1992) we have calibrated the extra-galactic Strömgren colors in terms of global evolutionary aspects. We have binned the galaxies into four classes - E/S0s, spirals, Seyferts and starbursts. We borrow the morphological terms E, S0 and spiral only to label the categories of star formation history common for these types, and caution that the observations reported here are by no means adequate to make any true morphological statement.

Cluster galaxies are assigned to one of the four "spectromorphological" classes by finding the template galaxy with the best matching colors, taking into account observational errors via a tolerance parameter $\sigma$. The efficiency of this method depends on the catalogue size, accuracy of the observations and the intrinsic scatter among individual galaxies of the same Hubble type. The classes are well separated: starburst galaxies deviate strongly from the trend of normal and Seyfert galaxies in the $m z$ versus $(b z-y z)$ diagram (see Rakos et al. 1996, Fig. 4). They all lie below $m z=-0.2$. This can be ascribed to the bimodal distribution of stellar colors in a starburst galaxy, intrinsic reddening combined with a starburst and possibly low metal content. Seyfert galaxies are distinguished by their very small $u z-v z$ and $m z \sim 0$ values in the $m z$ vs. $u z-v z$ diagrams (Fig. 4 of Rakos et al. 1996), the photometric translation of a power-law SED.

\subsection{Estimating physical parameters}

The reddening of individual stars can be estimated using reddening-free parameters introduced by Strömgren. Independently, we have shown that intrinsic reddening can be determined separately from age and metallicity effects for galaxies as well, based on population synthesis models (Steindling et al. 2001). For example, the intrinsic reddening vector in $b z-y z$ vs. $m z$ or $u z-v z$ vs. $m z$ diagrams is perpendicular to the Hubble sequence (Rakos et al. 1996). Thus we can eliminate this variable from further analyses. Once the effect of reddening is removed, the color indices depend mostly on the age of the last starburst and on the total mass involved in it. This dependence can be calibrated using theoretical models of starburst galaxies (Rakos \& Schombert 1999). 
Table 1. A2218 cluster population

\begin{tabular}{llll}
\hline Type & Blue & Red & Total \\
\hline E/S0 & 5 & 25 & $30(77 \%)$ \\
Sp/Irr & 3 & 2 & $5(13 \%)$ \\
Seyfert & 0 & 2 & $2(5 \%)$ \\
Starburst & 1 & 1 & $2(5 \%)$ \\
\hline Total & $9(23 \%)$ & $30(77 \%)$ & 39 \\
\hline
\end{tabular}

It is worth mentioning how the amplitude of the $4000 \AA$ break $[D(4000)]$ in spectra of galaxies is related to the $u z-v z$ color index. A comparison of the $D(4000)$ values of Dressler \& Shectman (1987) for the two dozen galaxies in common with our nearby galaxy sample reveals a linear relation:

$$
2.5 \log D(4000)=0.646(u z-v z)+0.245
$$

with a correlation coefficient of 0.91 (the sample contains many types of galaxies). For individual stars, $u z-v z$ correlates with effective temperature, surface gravity, and metallicity. However, for an integrated stellar population, it is primarily a temperature indicator (Hamilton 1985). Thus the amplitude of the break is particularly sensitive to galaxy evolutionary effects.

\section{Results}

\subsection{Analysis of the Strömgren data}

Photometry in all four filters has been carried out for 108 objects. With a tolerance level of $\sigma \leq 0.13$ being representative of our photometric accuracy, a total of 39 cluster members has been identified.

For clusters at this particular redshift, the star and interloper rejection mechanism works so well, that we expect only 0.10 stars and effectively 0.0 field galaxies to remain unidentified within the magnitude range of our observations!

\subsubsection{Population statistics}

The population fractions are listed in Table 1 . The reader is cautioned again that this classification scheme is based on integrated colors and is not a morphological system. The designation of E/S0, Sp/Irr, or Starburst refers only to the current star formation rate and recent SF history of the galaxy, not to its morphological appearance, the existence of spiral arms, or a bulge to disk ratio.

We find that $30(77 \%)$ of the cluster members are E/S0, whereas $5(13 \%)$ are Sp/Irr, $2(5 \%)$ are starburst and the remaining $2(5 \%)$ are classified as Seyfert galaxies. Typical E:S0:Sp/Irr ratios for present day clusters are $20 \%: 40 \%: 40 \%$, based on morphological classification of nearby cluster galaxies (Oemler 1992). The cluster A2317 $(z=0.211)$, at a redshift similar to A2218, has been observed in a similar manner, but on a larger telescope yielding deeper images of a slightly smaller field of view

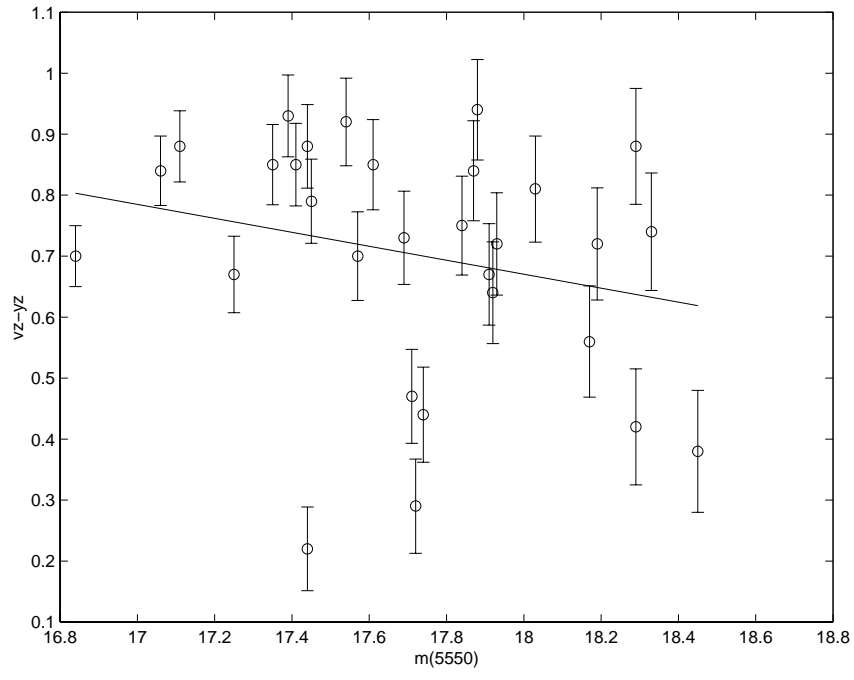

Fig. 1. Color-magnitude relation for the E/S0 population

(Rakos et al. 1997). It was found to be $20 \%$ overabundant in late type galaxies as compared with present day clusters. (For the comparison to present-day cluster population statistics it is assumed that all the galaxies photometrically classified as $\mathrm{Sp} / \mathrm{Irr}$, starburst and Seyferts would be contained in the morphological late type ( $\mathrm{Sp} / \mathrm{Irr}$ ) category.) From this point of view, A2218 is even more evolved than present day clusters. We believe that this "development speed" is produced by the remarkable cluster density.

The large number of Seyfert galaxies in A2218 (5\%), on the other hand, is very similar to the number found in A2317 and to the fraction of AGN galaxies in 3C 295. In contrast, the fraction of Seyferts found in local clusters is extremely low, less than $1 \%$. However, a recent study by Sarajedini et al. (1996) finds the fraction of AGNs in the field to be $10 \%$ for $z=0.2-0.6$. One might argue that the reliability of the quoted $5 \%$ is rather weak due to small number statistics, but this is in fact a lower limit. As we have shown in Steindling et al. (2001), our method does not necessarily identify every AGN present due to inclination effects, but if an object is identified as hosting an AGN, this identification is very reliable. Thus, our value of $5 \%$ is in line with the increase of AGN activity at these redshifts in both cluster and field environments, suggesting that the AGN increase is independent of environment.

\subsubsection{Color-magnitude relation}

The C-M relation for single burst stellar populations, such as ellipticals and S0s, is a reflection of the massmetallicity correlation predicted by simple closed-box models of galaxy evolution (Faber 1973; Tinsley 1980). The greater the mass of a galaxy, the higher its luminosity and its capacity to retain SN-driven wind-material, resulting in a more rapid enrichment of the first generation of stars and, therefore, a redder red giant branch for the composite population. The $v z$ filter is located over several strong metal lines in an old population spectrum and $b z$ 
samples the red continuum in a region free of any emission or absorption lines, thus the $(v z-y z)$ color is sensitive to metallicity changes. Figure 1 presents the $(v z-y z)$ color-magnitude diagram for the E/S0 population. Despite the large scatter, a trend of redder metallicity color with higher luminosity, or rather mass is visible. The leastsquare linear fit to the data is also shown (solid line). The effect is comparable to what we observed in A2317. According to Visvanathan \& Sandage (1977), the slope of the color-magnitude relation is nearly zero from $7000 \AA$ to $5000 \AA$ and increases steadily shortward of $4300 \AA$, reaching a maximum value of $0.11 \mathrm{mag}$ per mag near $3400 \AA$. For small redshifts this is universal and independent of the galaxies distance and the density of its environment. This translates into a blueing in $(v z-y z)$ of 0.06 mag per mag. The best fit linear regression of the data points in Fig. 1 is found to be:

$(v z-y z)=(2.73 \pm 0.59)-(0.11 \pm 0.04) m(5550)$

in agreement with the predicted value.

\subsubsection{The Butcher-Oemler effect}

The original broadband definition of the fraction of blue to red galaxies, $f(B)$, given by Butcher \& Oemler (1984), is the ratio of galaxies $0.2 \mathrm{mag}$ bluer in $(B-V)$ than the mean color of the E/S0 sequence (after k-corrections) to the total number of galaxies in the cluster. We have defined the fraction of blue galaxies as the ratio of the number of galaxies bluer than $(b z-y z)=0.22$ to the total number of galaxies. Since the mean $(b z-y z)$ color of present day ellipticals is 0.37 (Schombert et al. 1993) and $(b z-y z)$ maps into $(B-V)$ in a linear fashion with a slope of 1.33 , a cut at $(b z-y z)=0.22$ is effectively the same as Butcher \& Oemlers 0.2 mag selection, and the two values are directly comparable.

Applying this criterion to A2218 produces an $f(B)$ value of $23 \%$. This value is similar to A115 (at the same redshift), but not to $\mathrm{A} 2317$ with an $\mathrm{f}(\mathrm{B})=35 \%$ (Rakos et al. 1997). The exact morphological type of the brightest blue galaxies cannot be determined from our data (our photometric classification does not distinguish between early and late type spirals), but results from recent HST imaging of distant clusters suggest that these systems are structurally late-type spirals with slightly increased levels of star formation activity (Oemler et al. 1997). With this in mind, it appears that paucity of spirals in A2218 partly causes the low $f(B)$ fraction.

Moreover, the deep rest frame four color photometry of A2317 (Rakos \& Schombert 1999) shows that the ratio of blue to red galaxies has a strong dependence on absolute magnitude, such that blue galaxies dominate the regions of very brightest and very faintest galaxies. We have also shown that the blue fraction of faint galaxies contains more starbursting galaxies $(m z \leq-0.2)$ than the bright blue galaxies. In the case of A2218 our observations are not deep enough to test for this effect. This can explain the small number of starburst galaxies in A2218. A two

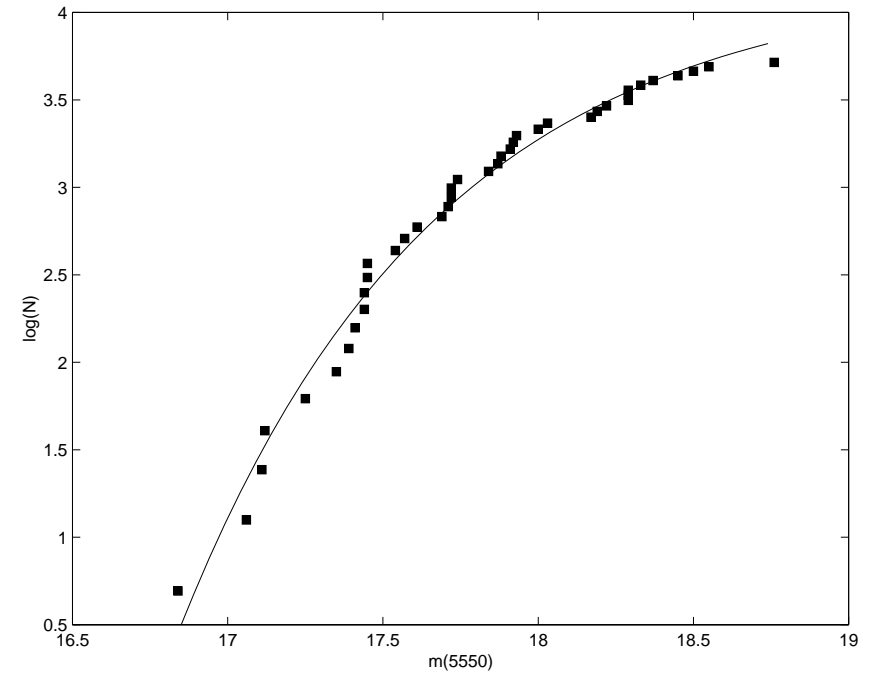

Fig. 2. Cumulative luminosity function. The solid line represents the best exponential fit $(A \exp (-B m(5550)+C))$ to the data points (filled squares)

magnitude deeper observing limit (a task for a $4 \mathrm{~m}$ class telescope) would be required to probe far enough beyond the knee in the luminosity function to reach the bulk of the dwarf galaxies and verify this issue. More evidence for the important role of dwarf galaxies can be found in the literature: deeper HST observations of $z \sim 0.3$ clusters by Koo et al. (1997), Oemler et al. (1997) and Couch et al. (1998) have shown that the most spectacular starbursts tend to be low mass objects, whose final state is likely to be that of a dwarf galaxy. Further support for this view comes from the observation that dwarf galaxies in Virgo, Fornax and Coma have undergone recent episodes of star formation. Optical luminosity functions for bright galaxies in these clusters are consistent with a Schechter luminosity function having an exponent around -1 but dwarf galaxies are better characterized by a power law with an exponent around -1.8. If we interpret Ferguson's (1993) $(B-V)$ color-magnitude relation for dwarf ellipticals in Virgo and Fornax in terms of the B\&O definition of "blue galaxies", the greater number of these dwarf ellipticals is blue. All this implies, that the Butcher-Oemler effect is strongly dependent on limiting magnitude.

Finally in Fig. 2 we present the luminosity function for A2218. It is very similar to the common luminosity function for bright galaxies. Deeper observations are required to study the dwarf population. Cluster galaxies are listed in Table 2, and a finding chart is provided in Fig. 3.

\subsection{Analysis of earlier observations}

One of the advantages of our observations is the large field of view available at Wise, which came at the cost of photometric depth. It is therefore useful to compare our results to those derived from other studies to verify that observational biases are correctly accounted for. We also want to link our results, derived from rather unusual 
Table 2. A2218 Cluster members. In Col. 7, $\sigma$ is the largest difference in color between the cluster galaxy and the most resembling template galaxy in the catalogue. Column 8 lists the color according to the B\&O definition, and Col. 9 our spectromorphological classification. Column 10 gives the number in Le Borgne's catalog, and Col. 11 the B\&O number

\begin{tabular}{|c|c|c|c|c|c|c|c|c|c|c|}
\hline $\mathrm{n} \#$ & $u z-v z$ & $b z-y z$ & $v z-y z$ & $m z$ & $m(5500)$ & $\sigma$ & color & type & Le Borgne ID & $\mathrm{B} \& \mathrm{O}$ ID \\
\hline 1 & 0.67 & 0.31 & 0.85 & 0.23 & 17.35 & 0.05 & red & $\mathrm{E} / \mathrm{S} 0$ & & 6 \\
\hline 2 & 0.75 & 0.30 & 0.88 & 0.28 & 18.29 & 0.08 & red & $\mathrm{E} / \mathrm{S} 0$ & & 44 \\
\hline 3 & 0.96 & 0.31 & 0.79 & 0.17 & 17.45 & 0.08 & red & $\mathrm{E} / \mathrm{S} 0$ & & 10 \\
\hline 4 & 1.09 & 0.33 & 0.70 & 0.04 & 17.57 & 0.08 & red & $\mathrm{E} / \mathrm{S} 0$ & 482 & 15 \\
\hline 5 & 0.98 & 0.27 & 0.56 & 0.02 & 18.17 & 0.05 & red & $\mathrm{E} / \mathrm{S} 0$ & 430 & 36 \\
\hline 6 & 0.83 & 0.32 & 0.70 & 0.06 & 16.84 & 0.05 & red & $\mathrm{E} / \mathrm{S} 0$ & 391 & 8 \\
\hline 7 & 0.48 & 0.38 & 0.84 & 0.08 & 17.06 & 0.07 & red & $\mathrm{E} / \mathrm{S} 0$ & 421 & 42 \\
\hline 8 & 0.45 & 0.38 & 0.67 & -0.09 & 17.25 & 0.09 & red & $\mathrm{E} / \mathrm{S} 0$ & 373 & 24 \\
\hline 9 & 0.65 & 0.35 & 0.92 & 0.22 & 17.54 & 0.09 & red & $\mathrm{E} / \mathrm{S} 0$ & 341 & 18 \\
\hline 10 & 0.52 & 0.38 & 0.75 & -0.01 & 17.84 & 0.05 & red & $\mathrm{E} / \mathrm{S} 0$ & 196 & 30 \\
\hline 11 & 0.64 & 0.30 & 0.88 & 0.28 & 17.11 & 0.05 & red & $\mathrm{E} / \mathrm{S} 0$ & 244 & 3 \\
\hline 12 & 1.00 & 0.37 & 0.73 & -0.01 & 17.69 & 0.05 & red & $\mathrm{E} / \mathrm{S} 0$ & 419 & 21 \\
\hline 13 & 0.97 & 0.38 & 0.72 & -0.04 & 17.93 & 0.08 & red & $\mathrm{E} / \mathrm{S} 0$ & 148 & 28 \\
\hline 14 & 0.83 & 0.42 & 0.94 & 0.10 & 17.88 & 0.05 & red & $\mathrm{E} / \mathrm{S} 0$ & 118 & 26 \\
\hline 15 & 0.60 & 0.32 & 0.85 & 0.21 & 17.61 & 0.05 & red & $\mathrm{E} / \mathrm{S} 0$ & & 19 \\
\hline 16 & 0.62 & 0.20 & 0.31 & -0.09 & 18.22 & 0.05 & blue & Sp/Irr & & 38 \\
\hline 18 & 0.21 & 0.35 & 0.62 & -0.08 & 18.37 & 0.08 & red & $\mathrm{Sp} / \mathrm{Irr}$ & & 49 \\
\hline 19 & 1.60 & 0.27 & 0.44 & -0.10 & 17.74 & 0.08 & red & $\mathrm{E} / \mathrm{S} 0$ & & 16 \\
\hline 20 & 1.16 & 0.10 & 0.43 & 0.23 & 18.50 & 0.13 & blue & $\mathrm{Sp} / \mathrm{Irr}$ & & 272 \\
\hline 21 & 1.05 & 0.34 & 0.88 & 0.20 & 17.44 & 0.11 & red & E/S0 & 630 & 11 \\
\hline 22 & 0.30 & 0.47 & 0.49 & -0.45 & 18.29 & 0.11 & red & burst & & \\
\hline 23 & 0.84 & 0.32 & 0.47 & -0.17 & 17.71 & 0.09 & red & $\mathrm{E} / \mathrm{S} 0$ & 113 & 17 \\
\hline 24 & 0.49 & 0.38 & 0.74 & -0.02 & 18.33 & 0.05 & red & $\mathrm{E} / \mathrm{S} 0$ & 77 & 36 \\
\hline 25 & 0.64 & 0.37 & 0.78 & 0.04 & 18.55 & 0.05 & red & Sy & & 54 \\
\hline 26 & 1.52 & 0.19 & 0.67 & 0.29 & 17.91 & 0.11 & blue & $\mathrm{E} / \mathrm{S} 0$ & & 27 \\
\hline 27 & 1.09 & 0.50 & 0.84 & -0.16 & 17.87 & 0.11 & red & $\mathrm{E} / \mathrm{S} 0$ & & 29 \\
\hline 28 & 2.16 & 0.17 & 0.29 & -0.05 & 17.72 & 0.09 & blue & $\mathrm{E} / \mathrm{S} 0$ & & 14 \\
\hline 29 & 1.38 & 0.16 & 0.07 & -0.25 & 17.72 & 0.09 & blue & burst & & 46 \\
\hline 30 & 0.89 & 0.34 & 0.81 & 0.13 & 18.03 & 0.05 & red & $\mathrm{E} / \mathrm{S} 0$ & & \\
\hline 31 & 0.68 & 0.27 & 0.54 & 0.00 & 18.00 & 0.05 & red & $\mathrm{Sp} / \mathrm{Irr}$ & & \\
\hline 32 & 1.14 & 0.28 & 0.85 & 0.29 & 17.41 & 0.11 & red & E/S0 & & \\
\hline 33 & 1.26 & 0.21 & 0.42 & 0.00 & 18.29 & 0.05 & blue & $\mathrm{E} / \mathrm{S} 0$ & & \\
\hline 34 & 0.80 & 0.33 & 0.72 & 0.06 & 18.19 & 0.05 & red & $\mathrm{E} / \mathrm{S} 0$ & & \\
\hline 35 & 2.14 & 0.22 & 0.38 & -0.06 & 18.45 & 0.05 & blue & E/S0 & & \\
\hline 36 & 1.03 & 0.09 & 0.12 & -0.06 & 18.76 & 0.13 & blue & $\mathrm{Sp} / \mathrm{Irr}$ & & \\
\hline 37 & 0.67 & 0.29 & 0.64 & 0.06 & 17.92 & 0.05 & red & E/S0 & & 25 \\
\hline 38 & 0.75 & 0.35 & 0.93 & 0.23 & 17.39 & 0.11 & red & $\mathrm{E} / \mathrm{S} 0$ & 235 & 9 \\
\hline 39 & 1.32 & 0.15 & 0.22 & -0.08 & 17.44 & 0.11 & blue & $\mathrm{E} / \mathrm{S} 0$ & & \\
\hline 40 & 0.73 & 0.31 & 0.53 & -0.09 & 17.12 & 0.05 & red & Sy & & \\
\hline
\end{tabular}

filters, to more classical photometric systems to make our results more accessible for further use.

\subsubsection{Photometric zero-point and accuracy}

The first photometry of A2218 was published by Butcher et al. (1983). The spectral range of the published data was similar to our $y z$ filter, thus we can compare our $m(5550)$ with their photometry (Fig. 4). The linearity and the scatter correspond to the accuracy of the good photographic photometry and the deviations cited in the aforementioned publication (0.15-0.25 mag).

More recently, Le Borgne et al. (1992) have carried out a detailed photometric and spectroscopic survey of the cluster. The photometry was performed in 5 bands, $B, g$, $r, i$ and $z$ for a sample of 729 objects. The spectroscopy concerns 66 objects. The derived rest frame velocity dispersion of $1370 \mathrm{~km} \mathrm{~s}^{-1}$ indicates a deep potential well. None of the galaxies identified as cluster members show emission lines. This is not in contradiction with our finding of 2 Seyfert-like galaxies and two starbursts, as these four objects are not part of Le Borgne et al.'s sample.

The authors have used several colors to show evidence for a strong color magnitude relation. The spectral type of galaxies was determined by fitting non evolved, redshifted, synthetic spectral energy distributions (Guideroni \& Rocca-Volmerange 1987) to the observed spectra. Further photometric analysis was not carried out. We reexamine the galaxies observed spectroscopically in 


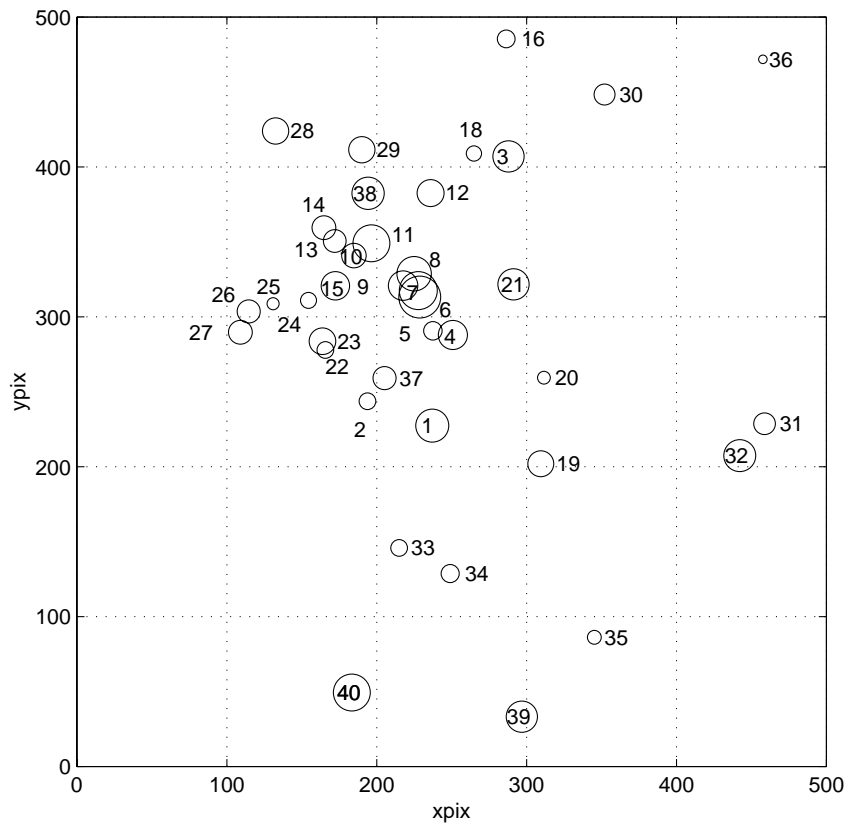

Fig. 3. Finding chart for cluster galaxies. The labels correspond to our ID numbers. The corresponding $\mathrm{B} \& \mathrm{O}$ and Le Borgne's ID numbers are given in Table 2

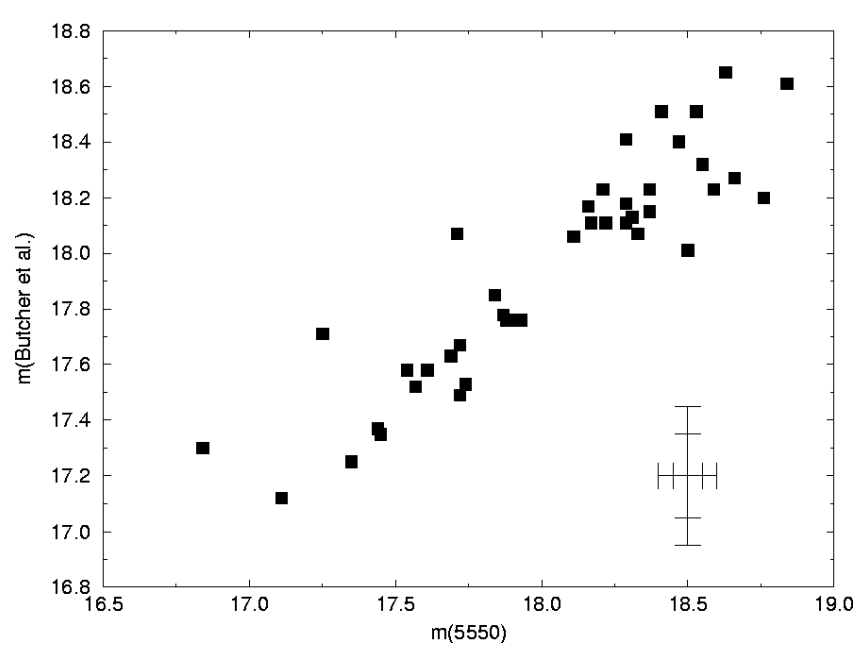

Fig. 4. Comparison between the magnitudes given by Butcher et al. (1983) and our $m(5550)$ magnitudes. Minimum and maximum error estimates are given in the lower right corner

what follows. Thirteen of our 40 cluster members are in common with the spectroscopic sample of Le Borgne et al. We compare our $m(5550)$ and their $r$ magnitudes in Fig. 5. The linear fit has a slope of 0.978 and presents very little scatter because both filters cover approximately the same spectral region and the color of these objects is within a very narrow range. In what follows, we analyze the full spectroscopic sample in the same way as our own Strömgren data to estimate selection biases.

\subsubsection{The Butcher-Oemler effect}

Eleven of the spectroscopic cluster members have been unequivocally classified as ellipticals by Le Borgne et al.

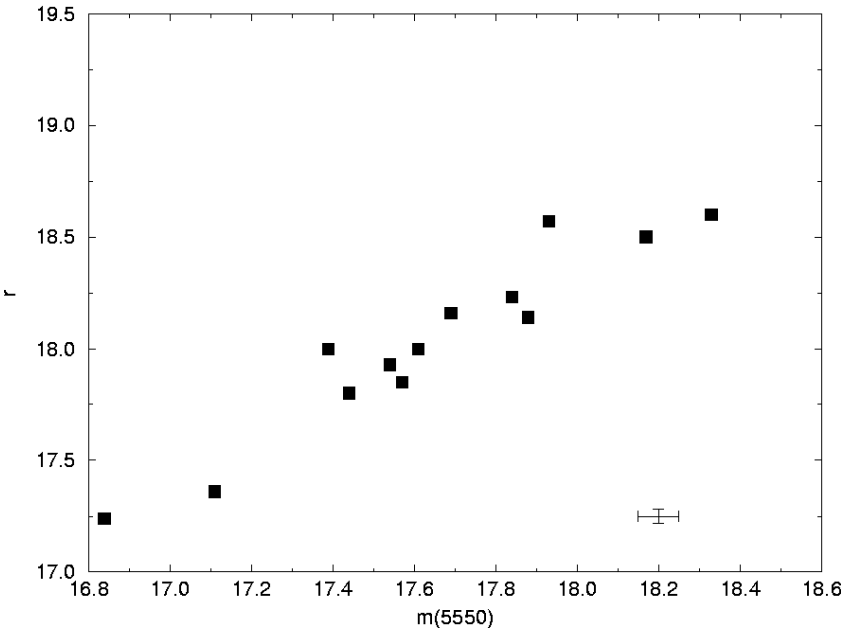

Fig. 5. Le Borgne's (1992) $r$ magnitude vs. our $m(5550)$

Their mean $(g-r)$ value is $0.92 \pm 0.16$. The relationship between $(g-r)$ and $(B-V)$ is linear with a slope near 1 (Thuan \& Gunn 1976).

$B-V=0.449+0.937(g-r)$.

Thus the Butcher-Oemler definition for blue translates into $(g-r) \leq 0.72$. At $z=0.171,(g-r)$ is very similar to $(b z-y z)$. Applied to the 41 spectroscopically observed galaxies whose redshift identifies them as cluster members, this relation yields only 5 blue galaxies, i.e., $f(B)$ is only $12 \%$ in contrast to $23 \%$ for our sample. The value of $12 \%$ is derived from a sample covering a central field of only $4^{\prime}$ by $4^{\prime}$, while our frame was $12^{\prime}$ by $12^{\prime}$. It is by now well-known, that blue galaxies avoid the cores of rich clusters, and it is therefore not surprising that we find a larger $f(B)$ with our significantly larger FOV.

\subsubsection{Color-magnitude relation}

We used the remaining 36 red galaxies to obtain the colormagnitude relation between $(B-r)$ and $r$ :

$B-r=2.998 \pm 0.622-(0.068 \pm 0.032) r$.

The $(B-r)$ color is very similar to $(v z-y z)$ at this redshift. The slope of 0.068 fits well in the range of the value derived from our brighter sample (0.089), indicating a continuity of the color-magnitude relation between giant and dwarf galaxies.

\section{Conclusions}

A2218 is an extremely compact galaxy cluster. The Butcher-Oemler process, seen in common clusters at various redshifts, has come to an end in A2218. The population statistics derived from our observations and the data published by Le Borgne et al. (1992) are the following: the fraction of blue galaxies is very small, within the cluster center only $12 \%$, and increasing to $23 \%$ further towards the outskirts. A deep spectroscopic survey of 
66 objects near the cluster center does not reveal even a single galaxy with emission lines (post starburst or AGN signature). Typical E:S0:Sp/Irr ratios for present day clusters are 20\%:40\%:40\% based on morphological classification of nearby cluster galaxies. The galaxies in A2218 are almost all of E/S0 type. More precisely, population fractions are: $77 \% \mathrm{E} / \mathrm{S} 0,13 \% \mathrm{Sp} / \mathrm{Irr}$, and $5 \%$ starburst galaxies (only two of our 39 members). This resembles Coma more than other $z \sim 0.2$ clusters, suggesting that the Butcher-Oemler effect is a signature of the clustering process influence on member galaxies, rather than intrinsic, cosmological galaxy evolution.

The remaining $5 \%$ of galaxies in A2218 - located outside the field-of-view of the spectroscopic sample - are classified as Seyfert, respectively AGN galaxies. This is in sharp contrast to the $<1 \%$ fraction found in present day clusters, but similar to the values we measured in other clusters at $z \sim 0.2$, and comparable to what is known about field galaxies in that redshift range. What was known to be true for isolated galaxies, that the AGN phenomenon is much more common at great distances, appears to be valid for cluster galaxies as well.

Acknowledgements. Financial support from Austrian Fonds zur Förderung der Wissenschaftlichen Forschung is gratefully acknowledged. Observations at the Wise Observatory are supported in part by a grant from the Israel Science Foundation. S. Steindling acknowledges support from the US-Israel Binational Science Foundation.

\section{References}

Abell, G. O., Corwin, H. G., \& Olowin, R. P. 1989, ApJS, 70, 1 Abraham, R., et al. 1996, ApJ, 471, 694

Almoznino, E., \& Brosch, N. 1998a, MNRAS, 298, 920

Almoznino, E., \& Brosch, N. 1998b, MNRAS, 298, 931

Ashby, M., Houck, J., \& Hacking, P. 1992, AJ, 104, 98

Bahcall, J. 1986, ARA\&A, 24, 577

Birkinshaw, M., \& Hughes, J. P. 1994, ApJ, 420, 33

Butcher, H., \& Oemler, A. 1984, ApJ, 285, 426

Butcher, H., Oemler, A., \& Wells, D. C. 1983, ApJS, 52, 183

Caldwell, N., \& Rose, J. A. 1998, AJ, 115, 1423

Couch, W., Barger, A., Smail, I., Ellis, R., \& Sharples, R. 1998, ApJ, 497, 188

Crampton, D., Le Fèvre, O., Lilly, S., \& Hammer, F. 1995, ApJ, 455, 96

David, L. P., Slyz, A., Jones, C., et al. 1993, ApJ, 412, 479

de Bruyn, A., \& Sargent, W. 1978, AJ, 83, 1257

de Propris, R., Pritchet, C. J., Harris, W. E., \& McClure, R. D. 1995, ApJ, 450, 534

Dickey, J., \& Lockman, F. 1990, ARA\&A, 28, 215

Donas, J., Milliard, B., \& Laget, M. 1995, A\&A, 303, 661

Dressler, A., \& Shectman, S. 1987, AJ, 94, 899

Dressler, A., Oemler, A., Butcher, H., \& Gunn, J. 1994a, ApJ, 430, 107

Dressler, A., Oemler, A., Sparks, W., \& Lucas, R. 1994b, ApJ, 435, L23
Driver, S. P., Phillipps, S., Davies, J. I., Morgan, I., \& Disney, M. J. 1994, MNRAS, 268, 393

Faber, S. 1973, ApJ, 179, 731

Ferguson, H. 1993, ESO/OHP workshop on Dwarf galaxies

Gallagher, J. S., \& Wyse, R. F. G. 1994, PASP, 106, 1225

Guideroni, B., \& Rocca-Volmerange, B. 1987, A\&A, 186, 1

Gunn, J., \& Oke, J. 1975, ApJ, 195, 255

Hamilton, D. 1985, ApJ, 297, 371

Held, E., \& Mould, J. 1994, AJ, 107, 1307

Heller, A., Almoznino, E., \& Brosch, N. 1999, MNRAS, 304, 8

Hogg, D. W., \& Phinney, E. S. 1997, ApJ, 488, L95

Kennicutt, R. 1992, ApJS, 79, 255

Kneib, J. P., Melleier, Y., Pello, R., et al. 1995, A\&A, 303, 27

Koo, D. 1986, ApJ, 311, 651

Koo, D., Guzman, R., Gallego, J., \& Wirth, G. 1997, ApJ, 487, L49

Le Borgne, J. F., Pello, R., \& Sanahuja, B. 1992, A\&AS, 95, 87

Lejeune, T., Cuisinier, F., \& Buser, R. 1997, A\&AS, 125, 229

Lejeune, T., Cuisinier, F., \& Buser, R. 1998, A\&AS, 130, 65

Lilly, S., Le Fèvre, O., Crampton, D., Hammer, F., \& Tresse, L. 1995, ApJ, 455, 50

Massey, P., Strobel, K., Barnes, J., \& Anderson, E. 1988, ApJ, 328,315

McHardy, I. M., Stewart, G. C., Edge, A. C., Cooke, B., \& Yamashita, K. 1990, MNRAS, 242, 215

Moore, B., Katz, N., Lake, G., Dressler, A., \& Oemler, A. 1996, Nature, 379, 613

Oemler, A. 1992, Clusters and Superclusters of Galaxies, ed. A. Fabian (Dordrecht: Kluwer), 666

Oemler, A., Dressler, A., \& Butcher, H. 1997, ApJ, 474, 561

Pello, R., Le Borgne, J. F., Sanahuja, B., Mathez, C., \& Fort, B. 1992, A\&A, 266, 4

Pello-Descayre, R., Soucail, G., Sanahuja, B., Mathez, G., \& Ojero, E. 1988, A\&A, 190, L11

Phillipps, S., Parker, Q. A., Schwartzenberg, J. M., \& Jones, J. B. 1998, ApJ, 493, L59

Rakos, K., \& Schombert, J. 1995, ApJ, 439, 47

Rakos, K., \& Schombert, J. 1999, in ASP Conference Series, The Evolution of Galaxies on Cosmological Timescales, ed. J. E. Beckman, \& T. J. Mahoney, in print

Rakos, K., Fiala, N., \& Schombert, J. 1988, ApJ, 328, 463

Rakos, K., Maindl, T., \& Schombert, J. 1996, ApJ, 466, 122

Rakos, K., Odell, A., \& Schombert, J. 1997, ApJ, 490, 194

Sandage, A., Binggeli, B., \& Tammann, G. 1985, AJ, 90, 1759

Sarajedini, V., Green, R., Griffiths, R., \& Ratnatunga, K. 1996, ApJ, 471, L15

Schombert, J., Hanlan, P., Barsony, M., \& Rakos, K. 1993, AJ, 106,923

Silk, J., Wyse, R. F. G., \& Shields, G. A. 1987, ApJ, 322, L59

Steindling, S., Brosch, N., \& Rakos, K. 2001, ApJS, 132, 19

Thuan, T. X., \& Gunn, J. E. 1976, PASP, 88, 543

Tinsley, B. 1980, Fundam. Cosmic Phys., 5, 287

Tyson, J. 1988, AJ, 96, 1

Visvanathan, N., \& Sandage, A. 1977, ApJ, 216, 214

Yamashita, K. 1995, in Clusters of Galaxies, ed. F. Durret, A. Mazure, \& J. T. T. Van (Éditions Frontières), 153

Yee, H., \& Oke, J. 1978, ApJ, 226, 52 